\title{
Healing process with the use of a new synthetic resorbable membrane
}

CLINICAL

INNOVATIONS
Hoornaert $\mathrm{A}^{*}$, Layrolle $\mathrm{P}^{* *}$,

* Faculty of Dental Surgery, CHU Nantes, France; ** Inserm UMR 1238, PHY-OS, University of Nantes, France

\section{Abstract}

Resorbable membranes are usually used in guided bone regeneration procedures. They present many advantages: no need for membraneremoval surgery; a wide range of surgical applications; a better costeffectiveness; and a decreased in patient morbidity. However, the difficulty of maintaining the barrier function for an appropriate length of time is considered a major drawback for resorbable membranes. In addition, the resorption process of the membrane may interfere with wound healing and affect the volume of regenerated bone.

- The objective of this study is to analyse the healing of soft tissue over a period from one to four weeks when using a synthetic resorbable membrane in various guided bone regeneration situations.

Ninety-three patients requiring a guided bone regeneration procedure were included in this study. A synthetic resorbable PLGA membrane with various bone substitutes were used in alveolar preservation, bone augmentation before implants or bone regeneration during implantation. After 1 and 4 weeks post-surgery, the early woundhealing index (EHI) was scored.

- After 1 week, a primary flap closure without exposure of the membrane was observed in $68 \%$ of cases. When the flap was well closed initially, the wound was completely healed in $86 \%$ of cases. At four weeks, $97 \%$ of cases had complete mucosal healing regardless of the initial situation.

This clinical study shows the safety and performance of a new synthetic resorbable membrane. When the membrane was initially exposed, it kept its integrity without loss of the biomaterial granules and without infection. In most cases, the complete soft tissue healing occurred at four weeks without problems.

\section{Background and Aim}

The use of resorbable membranes is a common practice for guided bone regeneration procedures. These membranes do not require a second surgery for removal and are used in a wide range of indications such as alveolar preservation, bone augmentation before or during implantation. However, most of the membranes have difficulty to maintain a barrier function when exposed.

The objective of this study is to analyze the healing of soft tissue over a period from one to four weeks when using a synthetic resorbable membrane in various guided bone regeneration situations.

\section{Methods and Materials}

Ninety-three patients requiring a guided bone regeneration procedure were followed in this study. The membrane used is a synthetic resorbable poly-lactic-glycolic acid membrane (PLGA, Tisseos $\AA$, Biomedical Tissues), together with various bone substitutes. Soft tissue healing was evaluated according to the criteria of the early wound-healing index (EHI), Wachtel, and All J Perio (2003).

The patients whose healing was evaluated in $\mathrm{EHI}$ of 1,2 or 3 were considered to have a primary flap closure those of the scores corresponding to EHI 4 and 5 were considered to have a secondary flap losure. At 8 days and 4 weeks post-operatively, comparisons were made between an initial exposure or non-exposure of the membrane after suturing, and as function of the type of intervention: alveolar preservation; bone augmentation before implantation; bone augmentation during implantation.

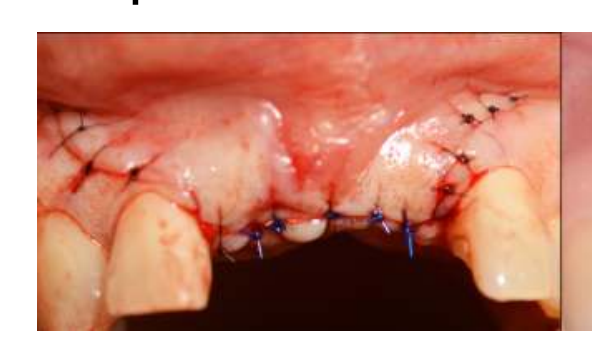

J0

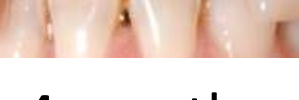

4 months primary flap closure
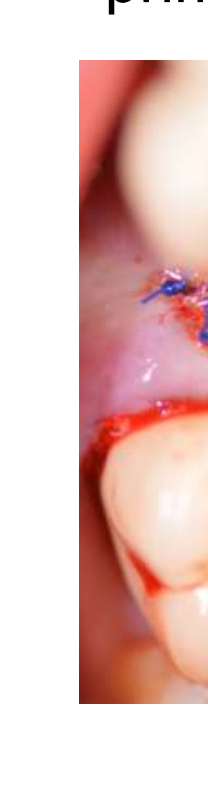

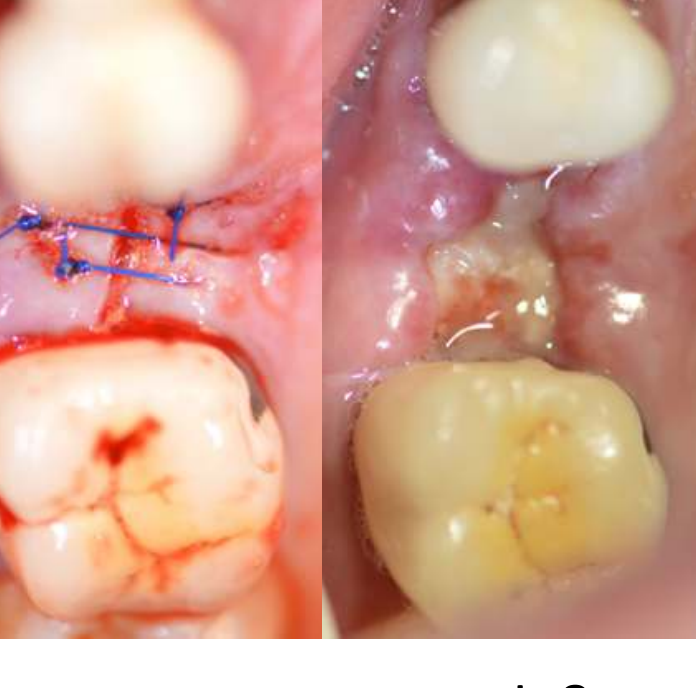

J0

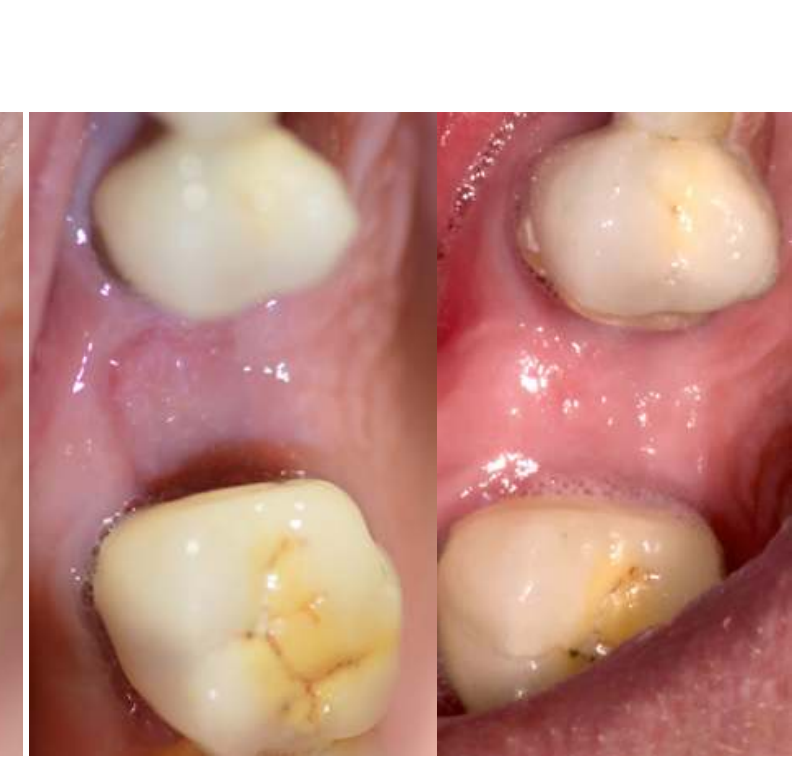

1 month

4 months
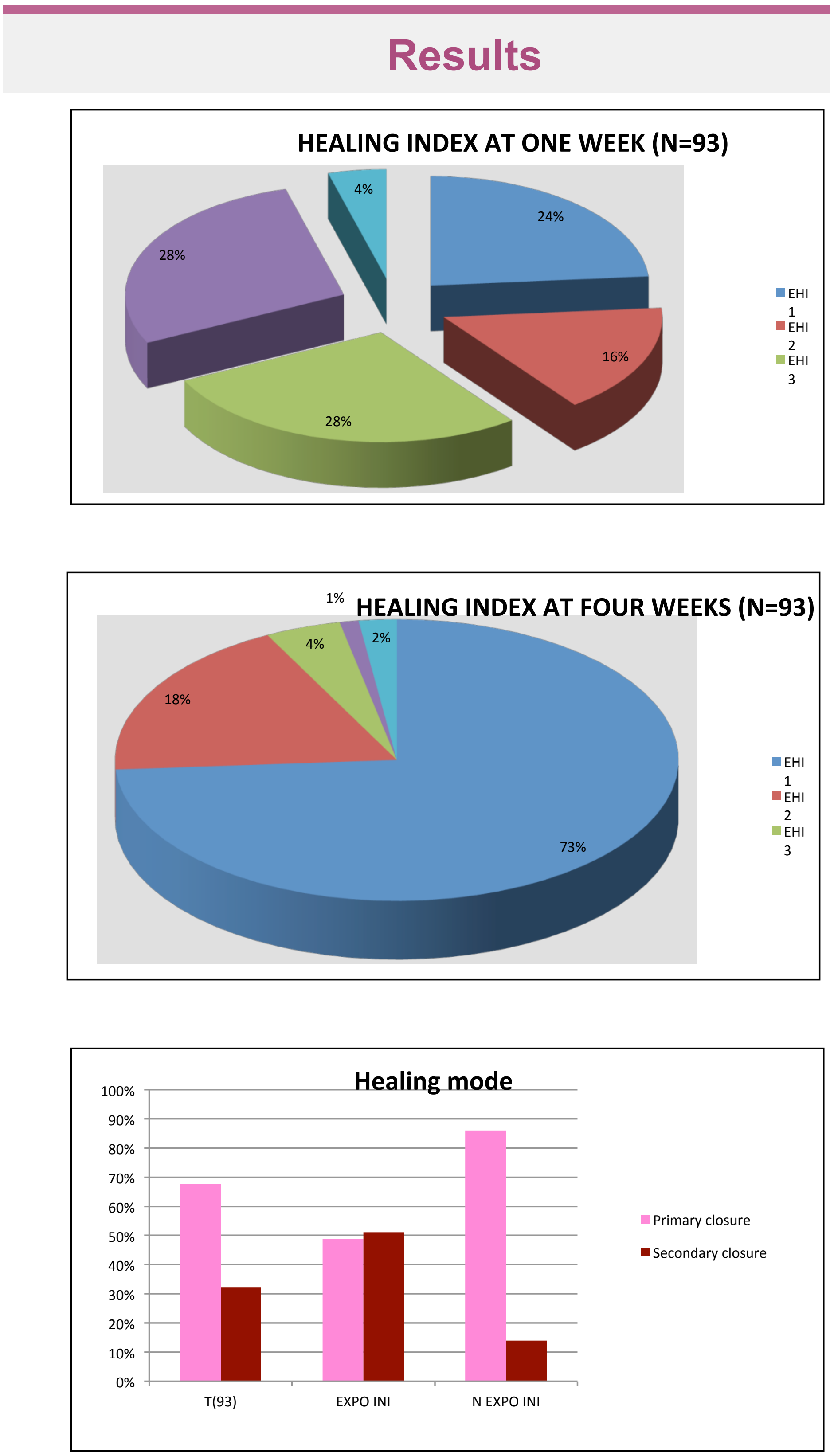

\begin{tabular}{|l|l|}
\hline $\mathrm{CH}^{2}=14,879$ & $\mathrm{P}=0,0001$ \\
\hline
\end{tabular}

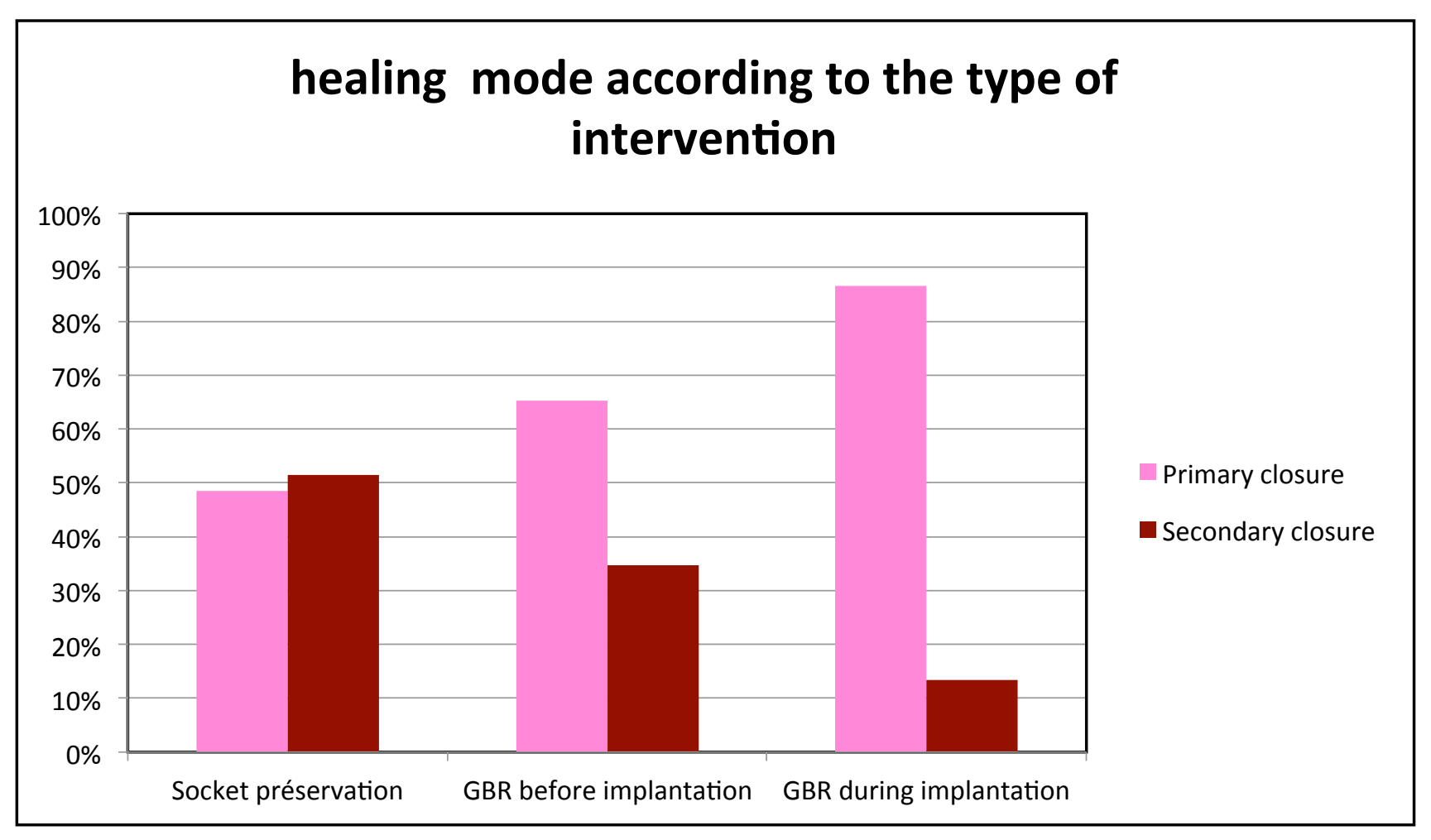

\begin{tabular}{|l|l|}
\hline $\mathrm{CHI}^{2}=10,266$ & $\mathrm{P}=0,0059$ \\
\hline
\end{tabular}

\section{Conclusions}

- This clinical study demonstrated the safety and performance of the new synthetic resorbable membrane. When the membrane was initial exposed, it kept its integrity without loss of the biomaterial granules and without infection. Re epithelisation of the gingival tissue was observed over the membrane. In most of cases, a complete soft tissue healing occurred at four weeks without major problems.

\section{References}

1. Wachtel H, Schenk G, Böhm S, Weng D, Zuhr O, Hürzeler MB. Microsurgical access flap and enamel matrix derivative for

. Hoornaert A D'Arros C. Heymann M-F Layrolle P. Biocompatibility, resorption and biofunctionality of a new synthetic biodegradable membrane for guided bone regeneration. Biomed Mater.2017

3. Moses O, Pitaru S, Artzi Z, Nemcovsky CE. Healing of dehiscence-type defects in implants placed together with different barrier membranes: a comparative clinical

Rosen PS, Reynolds MA. Guided bone regeneration for dehiscence and fenestration 